Istituto Fototerapico annesso alla Clinica Dermosifilopatica del R. Istituto di Studi Superiori in Firenze e diretto dal Professore Celso Pellizzari.

\title{
Histologische Untersuchungen über die Wirkung der Kromayerschen Quarzlampe auf die normale Haut des Menschen und beim Lupus vulgaris.
}

Von

\author{
Dr. Jader Cappelli, \\ Assistenten der Klinik.
}

(Hiezu Taf. V.)

Zwei Jahre sind erst kaum verflossen, seit die K romay ersche Quarzlampe in die dermatologische Therapie eingeführt wurde und schon ist über dieselbe eine sehr umfangreiche Literatur erschienen. Der Grund der zahlreichen, wissenschaftlichen Untersuchungen über ihre biologische und therapeutische Wirkung kann sicher nicht innerhalb der Grenzen der Errungenschaft eines neuen Apparates beschränkt bleiben; in der Tat bildet sie zu gleicher Zeit eine Frage von höchster, wissenschaftlicher und ökonomischer Bedeutung. Denn, abgesehen von den anderen therapeutischen Indikationen, für die sie ursprünglich ibr Autor empfahl, hat tatsächlich, nach dem Autor selbst, die Kromayer'sche Quarzlampe auf dem Gebiete der Lupusbehandlung das Problem gelöst, woran seine verschiedenen Vorgänger mit unsicherem Erfolge gearbeitet hatten, jenes Problem nämlich, das dahin geht, bei der Lupusbehandlung den originalen $F$ in s en-Apparat durch eine andere Vorrichtung mit denselben Qualitäten zu ersetzen, deren Aktuierung und praktische Diffusion aber zu gleicher Zeit auch leichter wären. 
Nach Kromayer (1) wären gegenüber anderen verschiedenen Lichtquellen die Vorzüge seiner Lampe folgende:

a) Kürzere Dauer der Bestrahlung.

b) Behandlung breiterer Flächen.

c) Leichte Installierung und Hantierung für den Arzt und Patienten.

d) Ersparnis an Stromkonsum.

Kr o m a y er fügte hinzu, daß, auf Grund seiner experimentellen Untersuchungen mit Chlor und Bromsilberpapier, seine Lampe an penetrierenden, aktinischen und aktiven Strahlen reicher resultierte als das Fin sen-Licht.

Nach der ersten Pablikation von $\mathrm{K}$ roma yer erschienen sofort einige eher ungünstige kritische Aufsätze von $W \mathbf{i} \mathrm{hm}$ ann (2) und SchüIer (3), die, trotz Anerkenaung einiger Vorzüge der Kro$m$ a y e $r$ schen Lampe, dieselbe in der Lupusbebandlung dem $F$ inse $n$ bogenlichte nicht gleich stellten. Dagegen verteidigte sich Krom a y e r (4) mit einem kurzgefaßten Artikel.

In der Versammlung der Berliner medizinischen Gesellschaft am 16. Januar 1907, zählte Blaschko (5) die Vorzüge und die Nachteile der Quarzlampe auf und erklärte ihre kurative Wirkung in der Lupusbehandlung für geringer als die des $\mathrm{F}$ ins en-Apparates. In einer dritten, im selben Monate des Jahres 1907 erschienenen Publikation behauptete $\mathrm{K}$ r om a yer (6) noch, daß seine Lampe bezüglich der Oberflächen- und Tiefenwirkung dem Finsen-Lichtbogen überlegen ist und lieferte einen Beitrag klinischer Untersuchungen, worunter vier Lupusfälle betreffen, auf die wir noch in kurzem zurückkommen werden.

Gegenüber den Behauptungen von Kromayer war es natürlich, daß man Kontrolluntersuchungen unternahm; so wurden zahlreiche experimentelle Untersuchungen angestellt, die überhaupt das Ziel verfolgten, einen Vergleich zwischen der Lampe von Kromayer und jener von $F$ insen herzustellen um dann zu entscheiden, ob man wirklich ihre Wirkung in der Lupusbebandlung als gleichwertig annehmen konnte.

Gunni Bask (7) nntersuchte komparativ die Wirkung der Kromayerschen und der Finsen Reyn-Lampe und kam hievei zu entgegengesetzten Schlußfolgerungen als $\mathrm{Krom}$ ayer. In der Tat erklärte er, daß „die Tiefenwirkung der Quarzlampe niedriger ist als jene der Finsen, um so niedriger je mehr Gewebsschichten zu penetrieren sind.“

Wichmann (8) machte zum Gegenstande seiner Studien die Wirkung der Emanationsstrahlen respektive aus der Kromayerschen und der Finsen Reyn-Lampe auf die menschliche Hant, nachdem diese Strahlen das Ohr eines Kaninchens penetriert hatten. Der Forscher zog den Schluß, daß „die spezifische biologische Wirkung des Finsen Reyn-Lichtes höher war als das weiße Licht der Kromayerschen Lampe". Der Autor schloß aber mittels des Methylenblau die Strahlen 
kleinster Wellenlänge aus; das Verhältnis invertierte sich gänzlich zum Vorteile der Kromayerschen Lampe, die in jeder Beziehung vorzuziehen war.

Emil Hesse und Karl Stern (9) behaupteten dagegen auf Grund ähnlicher Untersuchungen, daß „das Licht der Kromay e r schen Lampe reicher an oberfächlich wirkenden Strahlen und ärmer an chemischen Strahlen von Tiefenwirkung ist".

M a a s (10) gelangte auch $\%$ denselben Resultaten und erklärte, daß die Tiefenwirkung der Quarzlampe viel niedriger als die des Finsen Reyn ist; dagegen ist die Oberflächenwirkung irritierend.

Rudolf Pürckhauer (11) untersuchte die vom weißen und blauem Lichte (mit Hinzufügung von Methylenblau) der Kromayerschen Lampe am Kaninchenohre produzierten histologischen Veränderungen.

Der Forscher zog den Schluß, daB das weiße Licht der Lampe „an der bestrahlten Partie eine Nekrose hervorruft, die größer ist als die vom therapeutischen Standpunkte aus erwünschte". Die Regenerationserscheinungen treten sehr bald auf, aber sie heilen manchesmal nicht vollständig die Störungen der Nekrose, wie bei gewöhnlichen Verbrennungen vorzukommen pflegt. Das blane licht produziert eine oberflächliche Nekrose, die jener des Finsen-Lichtes ähnlich ist; die Regenerationserscheinungen des letzteren sind $\mathrm{rapider}$ und vollständiger. Der Autor bemerkte, daßs seine Behauptungen sich auf das Kaninchenohr bezogen und legte sich bezüglich der menschlichen Haut Reserve auf.

Mulzer (12) nahm die vergleichenden experimentellen Untersuchungen über die Kromayersche und Finsen Reyn-Lampe noch auf und studierte die chemische und baktericide Wirkung solcher Lichter, die sie progressiv dickere Schichten animalischen Gewebes penetrierend ausüben. Die Folgerungen des Autors gipfelten darin, daß bezüglich der Oberflächenwirkung der zwei Lichtarten sich ein großer Cnterschied zwischen der Finsen und Kromayerschen Lampe nicht ergab; aber das blane Licht der letzteren bedarf trotz ihrer auffillenden größeren baktericiden Wirkung einer längeren Zeit, um auf ein lichtsensibles Papier im selben Grade zu wirken.

$\mathrm{Zu}$ denselben Resultaten beinahe gelangte, betreffs des Penetrationsvermögens durch dickere Gewebe, Busk (13) aus dem Finsenschen Institute zu Kopenhagen.

Wetterer (14) berichtet in seinem Resumé bezüglich der K roma yerschen Lampe über einige gute Resultate, die er in wenigen Fällen von Lupus and Verrucae erzielte und behauptet, „daß die Kromayersche Lampe gegenüber der Finsen einen nennenswerten Fortschritt bedeutet".

Johansen (15) aus dem Finsenschen Institute studierte ver. gleichend die Konstitution und Extension des Spektrums des Finsen und Kromayerschen Lichtes und fand, daß „das Kromayersche Licht wegen der Qualität der ultravioletten Strahlen höher ist als das 
Finsen Reyn, niedriger aber wegen der Quantität der Strahlen des sichtbaren Spektrums.

Behring (16) seinerseits stellte eine lange Serie von vergleichenden Untersuchungen über die verschiedenen Lichtarten und zog den Schluß daß „die Kromayersche Lampe; alle anderen, inbegriften die Finsen Rey n, bezüglich der chemischen Wirkung und des Penetrationsvermögens übertrifft".

In der letzten Zeit haben Bordier und Nogier (17) eine detaillierte Studie über die physikalischen, chemischen und biologischen Eigenschaften des Lichtes publiziert und ausgezeichnete therapeutische Eigenschaften bei verschiedenen Dermatosen, bosonders beim Lupus hervorgehoben.

Fast zu gleicher Zeit erschienen aus der Finsenschen Schule eine Arbeit von Maar und eine von Jansen.

Maar (18) studierte nenerdings mit zahlreichen und feinen Untersuchungen die Tiefenwirkung des Kromayerschen und Finsen Reyn-Lichtes an Kaninchenohren, indem er für beide Lichtarten dieselben indentischen Bedingungen zu erhalten versuchte." Der Autor behauptete, daß das Finsen Reyn-Licht fähig ist auf das lebende animalische Gewebe eine tiefere und dauerndere Wirkung als das Kromayersche hervorzurufen, das mit Hinzufügung von Menthylenblau kein Penetrationsvermögen erhält. Für eine Oberflächenwirkung wäre das Kromayersche Licht vorzuzieben, wenn es nicht leicht mit lebhaften Schmerzen begleitete Erscheinungen von Nekrose hervorrufen würde.

Jansen (19) untersuchte als letzter die vom Kromayerschen Lichte am Kaninchenohre produzierten histologischen Veränderungen. Der Autor hebt hervor, daB solche Alterationen einen ProzeB von "Photodermatitis“ bilden, welche en gros jener von Finsen ähnelt, obgleich Unterschiede in der Wirkungsweise beider Lampen bestehen. Das Kromayersche Licht produziert sehr ausgesprochene Destruktionsphänomene, während die Proliferationserscheinungen mit geringerer Beschleunigung und weniger üppig auftreten".

Der von uns kurzgefaßte angeführte Überblick der Literatur beweist, daß, beim gegenwärtigen Stande der Frage, wir nicht in der Lage sein können, uns einen deutlichen und präzisen Begriff von dem therapeutischen Werte der Kromayerschen Lampe zu machen. In der Tat untersuchten die meisten Autoren experimentell die chemischen und biologischen Eigenschaften der Lampe im Vergleiche zu dem Finsen-Apparate. Aber wie viele therapeutische Indikationen besonders bezüglich des Lupus können wir aus den betreffenden Resultaten ableiten? Es ist eine Tatsache, daß, trotzdem einige Autoren das Experiment, soweit als nur möglich, so 
anstellten, daß es sich den therapeutischen Applikationen näherte, dennoch, zwischen diesen und ersteren, essentielle Differenzen vorhanden sind, die uns, bezüglich der Schlußfolgerungen, eine große Reserve auferlegen. Tatsächlich, um nur die am lebenden animalischen Gewebe angestellten Untersuchungen zu zitieren, kann niemandem entgehen, welch großer Unterschied in den anatomischen und physiologischen Verhältnissen zwischen einem feinen Kaninchenohre und der vom Lupus befallenen menschlichen Haut besteht. Ersteres fein, zart, transparent, letztere dick, von einem granulomatösen, manchmal mit einer resistenten Masse von Bindegewebe umgebenen Gewebe infiltriert und tief in verschiedenen Geweben gelagert. Solche Zustände müssen die Modalität der Reaktion welche die Haut dem physikalischen und chemischen Reize des Lichtes darbieten muß, beeinflussen. Übrigens, sind einige Autoren die ersten, welche dies anerkennen und zuletzt Jan sen (20) behauptete zum SchluB seiner Arbeit, daß es der Klinik vorbehalten bleibt, das letzte Wort über die therapeutische Wirkung der Kromayerschen Lampe auszusprechen.

Überdies fehlen oder wurden, soweit uns bekannt ist, nicht publiziert methodisch ausgeführte klinische Untersuchungen über zahlreiche und verschiedene Lupusfälle. Die von einigen Autoren wenig zitierten Fälle, darunter die vom Kromayer, unterliegen leicht der Kritik. Vor allem wegen ihrer geringen Zahl, in zweiter Linie, weil sie früher größtenteils nach anderen Methoden, darunter auch mit dem Finsen-Apparat, behandelt wurden, schließlich weil in keinem einzigen Falle jemand die histologische Untersuchung zur sicheren Kontrolle der klinischen Erscheinungen unternahm.

Professor Pelliz zari (21) wies in seinem Berichte über die Phototherapie, den er dem internationalen Kongresse für physikalische Therapie in Rom erstattete, bei der Besprechung der verschiedenen Quecksilberdampflampen als Ersatz für den Finsen-Apparat, auf einige guten, mit der Kromayerschen Quecksilberdampflampen - womit in der letzten Zeit das von Pellizzari begründete Institut für Phototherapie in Florenz bereichert wurde, - erzielten Resultate hin und behielt sich vor, systematisch, sowohl klinische als auch histologische Untersuchungen mit der Kromayerschen Lampe, die zweifellos in kurzer Zeit das Primat als Ersatz für den Finsen-Apparat errang, anstellen zu lassen. 
Mazzoni (22), erster Arzt am Institute für Phothoterapie in Floren $z$, der mit dem klinischen Teile dieser Untersuchungen betraut wurde, berichtete darüber dem in San Remo im Mai 1908 tagenden italienischen Kongresse für Hydrologie, Klimatologie und physikalische Therapie. Nach einer Kritik der therapeutischen Eigenschaften der verschiedenen Quecksilberlampen kam der Autor zu dem Schlusse, daß alle, trotz des Reichtums an aktinischen Strahlen, oberflächlich wirken und die Kromayersche von allen einen merklichen, wenn auch gegenüber dem $\mathrm{F}$ insen-Apparate geringeren Fortschritt in der Lupusbehandlung darstellt.

In der Folge deutete Professor Pellizzari im summarischen Berichte über die Tätigkeit des Jahres 1907 auf den Vorzug der Wirkung der Kromayerschen Lampe gegenüber dem Finsen-Apparate öfters in Fällen von Lupus erythematodes hin, immer in der Überzeugung aber, dab bei der Behandlung des Lupus vulgaris der Finsen-Apparat überlegen ist. Die klinische Erfahrung des Vorstandes des Institutes für Phototherapie in Florenz würde also jene Forscher stützen, die den Schluß gezogen haben, daß die Kromayersche Lampe von mehr aktiver, aber oberflächlicherer und kürzerer, die Proliferationserscheinungen zu beeinflussen weniger geeigneter Wirkung, gegenüber dem FinsenApparat, ist.

Jedenfalls wïnschte Professor Pellizzari, wie er sich in seinem Berichte auf dem Kongresse in Rom ausgesprochen hatte, die methodische Fortsetzung der Untersuchungen an Patienten seiner Klinik und beauftragte uns, methodisch die Wirkung der Kromayerschen Lampe an der normalen Haut des Menschen und an der vom Lupus vulgaris befallenen zu untersuchen und die erzielten Resultate in Beziehung zu den in der Klinik selbst nach den Fins e n-Applikationen vorher ausgeführten Untersuchungen $\mathrm{zu}$ bringen.

Da die klinischen Resultate, welche durch die Behandlung mit der Kroma yer's chen Lampe erzielt werden, verschieden sind ron den nach der Methode von Finsen, so ist es logisch anzunehmen, daß die beiden Lichtarten auf die Haut einen verschiedenen Mechanismus ausüben. Es genügt nicht dazu die exakte und präzise Komposition der beiden Spektra, die Quantität der dieselben bildenden Strahlen und die einzelnen physikalischen und biologischen Eigentümlichkeiten der Strahlen selbst zu kennen; sondern es ist notig direkt die unmittelbaren und entfernten Wirkungen an der Haut, welche die „Gesamtheit" der Strahlen unter verschiedenen Umständen hervorzubringen fähig sind, zu verfolgen. 
Dies bildet den Gegenstand unserer vorliegenden Untersuchungen. Wir haben uns vorgenommen, das "histologische Substrakt" der Reaktion zu studieren, welche das Licht der K romayer schen Lampe an der normalen menschlichen Haut und an der lupösen zu bedingen die Fähigkeit hat.

Bezüglich der gesunden Haut wollten wir mittels an verschiedenen Terminen vorgenommener Biopsien die Natur, Entität und Dauer der durch die Lichtapplikationen produzierten Alterationen in den Geweben und die Rapidität und Extension der Reaktionserscheinungen der Gewebe selbst untersuchen.

Bei der lupösen Haut wollten wir sehen, ob der histologische Prozeß einer solchen Reaktion von dem an der normalen Haut hervorgebrachten verschieden wäre und das Maximum einer histologischen Reaktion wahrnehmen, das man durch eine wachsende Zahl ron Applikationen erreichen kann, um festzustellen, welche Analogien mit dem Heilungsprozesse bestehen könnten, den man nach der Methode von Finsen erzielt.

Wir benützten das große Material von Lupus, über welches unsere Klinik und das derselben angegliederte Institut für Phototherapie verfügen und konnten so, um unseren Untersuchungen einen größeren Wert zu verleihen, zwei absolut notwendigen Bedingungen entsprechen: $a$ ) unter den zahlreichen Fällen von Lupus vulgaris jene zu wählen, die die gewöhnlichsten Varietäten des Typus darstellten, den solche Form gewöhnlich klinisch annimmt: Lupus circumscriptus, Lupus tumidus, Lupus diffusus usw.: $b$ ) zum Zwecke des Experimentes einzig jene Fälle $\mathrm{zu}$ verwenden, von denen wir die Sicherheit hatten, daß sie vorher gar keiner Behandlung, weder chemischen, noch chirurgischen, noch phototherapeutischen unterzogen worden waren.

Wir bemerken, daß die Applikationen in einer verschieden $3 / 4-1$ Stunde dauernden Sitzung mit der Kromayerschen Lampe und Hinzutügung von Methylenblau und mit der größten mit der Struktur und Hantierung des Apparates verträglichen Kompression gemacht wurden.

Was die histologische Technik anbelangt, so asportierten wir bei jeder Biopsie sorgfältig mit der bestrahlten Haut 
ein kleines Stückchen von nebenliegender gesunder Haut, um besser die Grenzen der histologischen Veränderungen kontrollieren zu können. Sofort nach der Abtragung wurde das Stückchen Haut in Heidenhains Sublimat fixiert und, nach den gewöhnlichen raschen Übergängen, in Paraffin eingebettet. Um bei der Beschreibung der einzelnen histologischen Untersuchungen nicht Wiederholungen anzuführen, erwähnen wir bezüglich der verschiedenen Tinktionen gleich, daß wir die gewöhnlichen Kernfärbungen, Hämatoxylin, Hämallaun, Boraxkarmin, Saffranin, polychromes Methylenblau, für das Bindegewebe Eosin, Orange, van Gies on und van Hansen außer den speziellen Färbungen ron Unna-Pappenheim für die Plasmazellen, Weigert für das Fibrin, Unna-Tänzer und Livin i für die elastischen Fasern, und die speziellen Methoden von Unna, Pelagatti für die Degeneration des Kollagens und des elastischen Gewebes anwandten.

\section{Biopsien gesunder Haut.}

Erster Fall. Zehnhellerstückgroßes, gesundes Hautstūckchen, mit der Kromayerschen Lampe 1 Stunde bestrahlt.

Biopsie: Sechs Stunden nach der Bestrahlung.

Makrosk op i c ch: Das Hautstïckchen ist stark gerötet, warm $\mathbf{u}$. dolent. Histologische Untersuchung. Epidermis normal dick, mit allen ihren Schichten. Die Hornschicht und das Stratum lu ci d um unregelmäßig erweitert und abgeblättert, so daß sie areoläres Aussehen baben. Die malpigische Keimschicht ist kompakt, normal dick; nur an einigen Stellen geringe Dilatation der Interzellularrăume und beginnende vakuoläre Degeneration der Stachelzellen.

Im Papillarkörper konstatiert man als erste Tatsache diskretes Ödem, das die dünnen Bindegewebsfasern gedehnt hat, die übrigens sich mit saueren Substanzen gut tingieren lassen und keine deutlichen Degenerationsveränderungen zeigen.

Die kleinen oberflächlichen Blutgefäße sind alle dilatiert; die meisten davon blutgefüllt; um dieselben spärliche, aus polynukleären Lueukocyten und kleinen runden Zellen bestehende Infiltrationselemente.

In der ganzen $\mathrm{Cu}$ tis keine Spur von Fibrin. Keine Veränderung der Drüsen außer Dilatation und Überfüllung der interlobularen Gefäßchen. Das elastische Gewebe ist in der ganzen Cutis bis zu den Papillenspitzen vorhanden; man bemerkt aber Fragmentierung der oberflächlichsten Fasern mit einigen Degenerationszeichen (E lac in). Solohe Alterationen, interstitielles ödem, Dilatation der Gefäße und geringe perivasale Exsudation, reichen bis in die tiefen Lagen der Cutis, 
Zweiter Fall. Gesundes Hautstückchen, 1 Stunde mit der Kromayer schen Lampe belichtet.

Biopsie: Achtzehn Stunden nach der Belichtung.

Makroskopisch: Die Haut ist warm, intensiv gerötet, dunkelrot, ödematös und überragt die umliegende Haut.

Histologische Untersuchung. Man konstatiert einen fortgeschritteren Grad der pathologischen Veränderungen. In der an die Applikationsstelle anliegenden Hautportion hat die Epidermis normales Aussehen mit Beibehaltung der wellenförmigen epiảermo-kutanen Trennungslinie; keine Veränderungen außer einer geringen Dilatation der Interzellularräume in der Keimschicht und einigen Zeichen von vakuolärer Degeneration in den Stachelzellen. In der entsprecbenden Partie der Cutis geringes Ödem, Dilatation der Gefäße und leichte perivasale Exsudation. Gegen die belichtete Partie hin rasche Vermehrung der Veränderungen. Mit der schwachen Vergrößerung gesehen ist die Epidermis wie eine Leiste von gleichförmigem Farbentone und präsentiert deutliche Alterationen in allen ihren Schichten. A b blät terung und irreguläre Trennung der Hornschicht, des Stratum lucidum und der Körnerschicht, Loslösung der Keimschicht durch Bildung zahlreicher, mit roten Blutkörperchen und mit aus den oberen Lagen der $\mathrm{Cu}$ tis stammenden polynukleären Leukocyten gefüllter Lücken. Die Stachelzellen sind gedehnt, von einander getrennt, meistens deformiert, vacuolisiert; ihr Kern, in dem Tøile größerer Reaktion, absolut nicht mehr tingierbar.

In der $\mathrm{Cu}$ tis stellt die deutlichste Veränderung ein difuses Ödem dar, dessen Intensitätsmaximum in den oberflächlicheren Lagen einen guten Teil der Epidermis in der Form einer breiten Blase trennt. Intensivere Dilatation und Thrombose der GefäBe, reichlichere perivasale Exsudation, rote Blutkörperchen, polynukleäre Leukocyten und kleine runde Zellen mit stark tingierbarem Kerne. Kein Fibrin. An einigen Stellen einige Degenerationserscheinungen der Bindegewebsfasern und einige Häufchen hyaliner Degeneration (Unna-Pelagatti) in den GefäBwandungen.

Keine deutlichen Veränderungen in den Drüsen und Follikeln. Strukturelle Degeneration der elastischen Fasern, speziell in den oberen Schichten der belichteten Partie.

Dritter Fall. Gesundes Hautstückchen aus dem Rande einer sukzessiven nach einer Blase, infolge einer halbstündigen Applikation, aufgetretenen Abschilferung.

Biopsie: Sieben Tage nach der Applikation.

Makroskopisch: An der belichteten Partie ein etwa zwanzighellerstückgroßer, mit einem leichten geröteten Hofe umgebener und einer blutigen Kruste bedeckter Substanzverlust mit flachen, nicht erhabenen Konturen.

Histologische Untersuchung. An der den Rand des Substanzverlustes einnehmenden Schnittläche zeigt die Epidermis zahlreiche 
intra- und subkorneale Blasen, Dilatation der Interzellular- und Spalträume, trübe und vacuoläre Degeneration der Stachelzellen.

In der Kutis das gewöhnliche Ödem und kleine Hämorrhagien um die dilatierten Gefäße. Solche Alterationen sind fortschreitend gegen die belichtete Partie hin markierter, entsprechend welcher der $\mathrm{Cu}$ tis eine amorphe, aus mehreren Schichten, die inmitten von koaguliertem Serum rote Blutkörperchen, polynukleäre Leukocyten, kleine Rundzellen, einige Fibrinfasern, fragmentierte Kerne nekrotisierter Elemente und Stachelzellen in fortgeschrittener Nekrose enthalten, bestehender Masse aufliegt. In den tieferen Lagen Vermehrung der Zellen im Exsudate, welches in gleichförmiger Weise die oberflächliche Lage der Cutis einnimmt.

In den tieferen Schichten der $\mathrm{Cutis}$ nehmen die Exsudationserscheinungen rasch ab, trotzdem das Ödem, die Dilatation der Gefäße und die kleinen perivasalen Hämorrhagien bleiben. Nur an der Peripherie des Substanzverlustes bemerkt man einige Spuren von fibroblatischer Proliferation.

Das Maximum der Veränderungen des Gewebes liegt also im epidermo-papillaren Anteile.

Vierter Fall. Gesundes Hautstiuckchen aus dem Zentrum der schon geheilten Fläche des vorangehenden Falles.

Biopsie: Fünfundzwanzig Tage nach der Applikation.

Makroskopisch: Die Hant ist vollständig geheilt, braun-rosarot, konsistent.

Histologische Untersuchung. Die Epidermis hat auf Kosten aller ihrer Schichten an Dicke zugenommen. Deutliche Erscheinungen außer von Hyperkeratose und Parakeratose von Akanthose. In den Zylinderzellen der Basalschicht zahlreiche karyokinetischen Figuren.

In der $\mathrm{Cu}$ ti s noch geringes diffuses Ödem und aktive fibroblastische Proliferation; tatsächlich konstatiert man junge Bindegewebszellen in den verschiedenen Stadien ihrer Entwicklung bis in den tiefsten Lagen der Cutis, wo noch Dilatation der Gefäße vorkommt; dagegen sind in den obersten Lagen noch ziemlich reichlich die Exsudationselemente gemischt mit Bindegewebszellen (1. Fig.) in Proliferation.

In den oberen und mittleren Lagen der $\mathrm{Cu}$ tis, wo das Maximum der Nekrose ist, keine Spur vom elastischen Gewebe; nur in den tieferen Lagen finden sich irreguläre Ramifikationen von Fasern mit dentlichen chemischen und strukturellen Alterationen.

\section{Biopsien lupöser Haut.}

Fünfter Fall. Lupusvalgaris cum Tuberculis distinctis. Bautstäckchen, ein typisches, linsengroßes ITuberkel, das zum ersten Male $3^{1} / 4$ Stunden belichtet wurde.

Biopsie: Achtzehn Standen nach der Applikation. 
Makroskropisch: Die Haut ist um die belichtete Partie gerötet, im Zentrum in einer leichten adhärenten Kruste abgehoben.

Histologische Untersuchung. Die Epidermis ist in toto über eine Blase, welche längs der ganzen Extension des Schnittes verlauft, abgehoben. Die getrennten und in einer amorphen Masse von koaguliertem Serum, in der man rote Blutkörperchen, Leukocyten und Zellendetritus sieht, fragmentierten oberen Schichten fehlen. Die malpighische Schicht ist auch tief alteriert; sie bildet eine irreguläre vacuolenreiche Masse; die von einander getrennten deformierten Stachelzellen sind größtenteils tief degeneriert; in den geschwollenen entfärbten Kernen nur einige Chromatinkörner an der Peripherie. In der Blase finden sich zusammen mit Detritus von koaguliertem Serum rote Blutkörperchen, polynukleäre Leukocyten und Fragmente nekrotisierter Zellen.

In den oberen Schichten der $\mathrm{Cu}$ ti s fällt als erste Veränderung ein sehr markiertes Ödem auf. Die entfärbten dünnen Bindegewebsfasern sind stark dilatiert und bilden ein Retikulum mit breiten Maschen, in denen eine eher rare, aus extravasierten Erythrocyten, polynukleären Lenkocyten, Bindegewebszellen mit aufgeblähtem ovalen Kerne und aus kleinen Zellen mit intensiv tingierbarem Kerne bestehende Infiltration vorkommt. Die Blutgefäße enorm dilatiert, einige thrombosiert. Gegen die tieferen Schichten der Cutis hin nehmen solche Entzündungserscheinungen merklich $a b$, aber ohne vollständig zu verschwinden und man kann sie um die granulomatösen Herde bis in die Tiefe der Cutis verfolgen.

Der granulomatöse Herd bildet noch eine kompakte Masse, aber man konstatiert nicht wie nach Applikationen mit dem $\mathrm{F}$ insen-Lichte jene Erscheinungen intensiven Ödems, die an einigen Stellen die Vereinigung gelöst haben.

Um die Riesenzellen finden sich, mit geringsten Alterationen, in dichten Schichten Plasmazellen und kleine Rundzellen; nur an der Peripherie des Knötchens sind spärliche polynukleäre Leukocyten und einige jungen Bindegewebszellen.

Unter dem granulomatösen Herde ist keine Leukocytenexsudation vorhanden; dagegen ziemliches Ödem und Dilatation der Gefäße.

Die elastischen Fasern bilden rund herum um den grannlomatösen Herd, in dem sie vollständig fehlen, ein Netz mit fragmentierten Maschen.

Sechster Fall. Lupus vulgaris cum magnis tuberculis dist in ctis. Hautstückchen mit einem linsengroßen Tuberkel, das 1 Stunde belichtet wurde.

Biopsie: Drei Stunden nach der Applikation.

Makroskopisch: Gerötete mit einer gelblichen adhärenten Kruste bedeckte Effloreszenz.

Histologische Untersuchung. Veränderungen ähnlich denen des rorerwähnten Falles. Akzentuierter sind die nekrotisch-degenerati- 
ven Erscheinungen der fast vollständig zerstörten Epidermis und der oberen Lagen der Cutis (Fragmentation und Dehnung der weniger tingierbaren Fasern).

Weniger ausgesprochen sind dagegen die Exsudationserscheinungen, die in den tiefen Lagen der Cutis fast vollständig fehlen.

Die elastischen Fasern verfolgt man bis in den Papillarkörper, wo sie fragmentiert und zum Teile degeneriert sind.

Im tieferen Teile des tuberkularen Herdes keine deutlichen Veränderungen, im oberen geringes Ödem mit spärlichen Exsudationserscheinungen.

Siebenter Fall. Lupus vulgaris cum parvis nodulis distinctis. Hautstückchen mit einem unversehrten kukuruzkorngroßen Tuberkel, das 1 Stunde belichtet wurde.

Biopsie: Vier Tage nach der Applikation.

Makroskopisch: Die Haut der Effloreszenz ist intensiv gerötet, in kleinen Blasen abgehoben.

Histologische Untersuchung. Die Epidermis vollständig in Blasen abgehoben und zum Teile von der $\mathrm{Cutis}$ getrennt; die Zellen größtenteils degeneriert, zum Teile nekrotisiert. Die Epidermis ist ferner von einer aus der $\mathrm{Cu}$ tis stammenden, entzündlichen Infiltration durchdrangen, in den oberen Lagen der Cutis dieselben Erscheinungen von Ödem, Exsudation, Dilatation der Gefäße und Thrombose.

Bemerkenswert vor allem reichliche, bis in die tiefen Lagen der Cutis diffuse Hämorrhagien, wo übrigens die wirklichen und eigenen Exsudationserscheinungenweniger akzentuiert sind. Die tuberkuiären Herde sehen immer eher kompakt aus und es scheint, dab sie wenig von den umliegenden pathologischen Veränderungen berührt wurden. Die Riesenzellen mit ibrem körnigen Protoplasma und ihren an der Peripherie geordneten Kernen sind nur im höchsten Teile des Knötchens, leicht infolge des Ödems, und des dichten Kranzes rom Plasma and lymphoiden Zellen, die sie umgeben, getrennt. Die kleinen Gefäße des granulomatösen Gewebes sind auch dilatiert und haben rund berum eine geringe Exsudation polynukleärer Leukocyten, die sich zwischen den Elementen des Gewebes spärlich mischen.

Die Haarfollikel zeigen geringe Dilatation der Wandungen und einige Infiltrationselemente im Innern.

Unbedentend sind die Veränderungen der Drüsen.

Das elastische Gewebe bildet um das Knötchen, in dessen Innern es vollständig fehlt, ein feines fragmentiertes Retikulum.

Achter Fall. Lupus vulgaris cum magnis tuberculis distinctis. Hautstückchen mit einem weizenkorngroßen Tuberkel, das $\%$ Stunden belichtet wurde.

Biopsie: Acht Tage nach der Applikation.

Makroskopisch: Die Applikation hat sukzessiv Rötung, Blase und Substanzverlust hervorgerufen; gegenwärtig ist letzterer auf dem 
Wege der Heilung. An den Rändern bemerkt man die Regeneration des neuen Epithels.

Histologische Untersuchung. Die Epidermis ist nur im periphersten Teile des Schnittes vorhanden; im Zentrum des Substanzverlustes feblt sie und die Cutis liegt entblößt.

Die ein wenig verdünnte Epidermis mit kaum ausgesprochenen welligen Leisten zeigt Erscheinungen von Parakeratose, noch akzentuiertes ödem der Keimschicht und Proliferationstätigkeit der Zylinderzellen der Basalschicht.

In den obersten Lagen der Cutis bleiben noch die Ektasie, Thrombose der GefäBe, die Hämorrhagien, das Ödem, diegemischte Exsudation polynukleärer Leukocyten und kleiner Rundzellen. Solche Veränderungen nehmen stufenweise an Intensität $a b$, so dab in den tieferen Lagen nur das ödem und die Ektasie der leeren Gefäße, deren aufgeblähte Endothelien innerhalb des Lumen hervorragen.

Bezüglich des Gewebes der tuberkularen Herde stellt man einige Zeichen von Vakuolisation und kleinzelliger Inklusion im Körper der oberflächlichsten Riesenzellen, welche das Ödem von den sie umgebenden Zellen getrennt hat. Um die oberflächlichsten Zellen spärliche Zeichen von Bindegewebsproliferation; hie und da Inselchen jungen Gewebes neuer Bildung mit oblungierten und linearen Kernen, aber keine aktive und diffuse neue Bildung.

Die Drüsen sind absolut unversehrt; die Knäuel vollständig regulär; die Epithelien regulär mit Protoplasma und normalem Kerne ohne Spuren von Infiltration um die Knäuel.

Das elastische Gewebe fehlt vollständig in der ganzen Extension des granulomatösen Gewebes; an der Peripherie nur dünne, bis zu den Papillen reichende Fibrillen desselben.

Neunter Fall. Lupus vulgaris circumscriptus cum dis tinctis tuberculis. Hautstückchen mit einem unversehrten Tuberkel, das 50 Minuten bestrahls wurde.

Biopsie: Zehn Tage nach der Applikation.

Makroskopisch: Der nach der Blase sukzessive Substanzverlust ist vollständig geheilt. Im Zentrum, im Gebiete eines Durchmessers von einem Zentimeter, ist das Epithel noch nicht reformiert.

Histologische Untersuchung. An der Epidermis fast dieselben Veränderungen wie im vorangehenden Falle.

In der $\mathrm{Cutis}$ noch im markierten Grade die gewöhnlichen Alterationen, Ödem, Ektasie, Thrombose der Gefäße und Exsudation erhalten. Nur spärlicher im Exsudate die polynukleären Leukocyten, welche vor allem in der perivasalen Partie bleiben, wo zahlreicher auch die Bindegewebszellen in Aktivität sind. Solche Zellen sieht man an der Peripherie des tuberkularen Herdes und nur einige Exemplare im Innern desselben. 
Man sieht nicht, wie nach derselben Zeit mit der Applikation des Finsen-Lichtes eineaktivefibroblastische Proliferation, mit der Tendenz sich in das granulomatöse Knötchen hineinzuschleichen. Für jetzt sind die Proliferationserscheinungen spärlich nnd wenig lebhaft.

Spärlich sind die Veränderungen der Riesenzellen, der Haarfollikel und der Drüsen.

Die elastischen Fasern, ein wenig fragmentiert, umfassen das ganze granulomatöse Knötchen und gelangen bis zur unteren Grenze des Papillarkörpers.

Zehnter Fall. Lupus vulgaris diffusus. Hautstückchen entnommen ans einer Effloreszenz am Ellenbogengelenke, die nur e in mal 45 Minuten belichtet wurde.

Biopsie: Zwanzig Tage nach Applikation.

Makroskopisch: Die Applikation hat eine lebhafte Reaktion (Rötung, Blase, Substanzverlust) hervorgerufen, die vollständig geheilt ist.

Die Haut ist glatter und scheint weniger infiltriert zu sein.

Histologische Untersuchung. Die Epidermis präsentiert Erscheinungen von Parakeratose, Hyperkeratose und Akanthose. In der Cutis persistiert noch $\mathrm{das}$ ödem und die Dilitation der GefäBe.

Die entzündliche Infiltration ist sehr spärlich, speziell bezüglich der polynukleären Leukocyten.

Zwischen den Elementen des Exsudates, diffus fast durch die ganze Extension der $\mathrm{Cu}$ tis in kleinem perivasalen Häufchen, sind die in der Cutis und an der Peripherie des tuberkulären Knötehens zerstreuten Fibroblasten. Das tuberkulöse Knötchen besteht aus spärlichen nicht alterierten Riesenzellen, um die sich Häufehen typischer Plasmazellen finden, welche die Tendenz haben sich in Ketten längs der Lymphgefäße zu diffundieren.

Dagegen keine Herdesehraktiver bindegewebiger Neubildung.

Keine bemerkenswerte Veränderungen der Drüsen.

Das elastische Gewebe ist in der Cutis sehr deutlich; in den oberflächlichen Lagen der Cutis nur in der Form kleiner, dünner fragmentierter granulöser Fibrillen vorhanden.

Elfter Fall. L u p u s vulg aris t u midus. Hautstückchen entnommen aus einer Effloreszenz von Lupus vulgaris tumidus, welche innerhalb von zwei Monaten sechsmal belichtet wurde; die Applikationen wurden nach dem Verschwinden der nach der vorangegangenen Applikation aufgetretenen Reaktion gemacht.

Die Effloreszenz ist weniger prominent und infiltriert.

Biopsie: Sechs Stunden nach der letzten Applikation.

Histologische Untersuchung. In der Epidermis alle Schichten in Blasen umgewandelt, eine Degeneration und an einigen 
Stellen eine. wirkliche und eigene Nekrose der getrennten, vollständig farblosen und fragmentierten Zellen der Keimschicht.

Solche Erscheinungen von Nekrose sind nooh in den obersten Lagen des Bindegewebes, wo zwischen den vom Ödeme enorm gedehnten fibrillären Maschen sich mit den extravasierten roten Blutkörperchen und polynukleären Leukocyten zahleiche, entfärbte und fragmentierte Zellkerve finden. Die entzündlichen Erscheinungen, die Dilatation der Gefäße und die Exsudation sind auch sehr intensiv und befallen auch den höchsten Teil der bis zum Papillarkörper hervorragenden tuberkulösen Knötchen. Hier präsentieren die vom granulomatösen Gewebe getrennten zahlreichsten Riesenzellen, mit den an einem Pole gedrängten Kernen, in ihrem Innern Zeichen von Vacuolisation.

Gegen die tieferen Lagen der Cutis zeigen die Riesenzellen keine deutlichen Veränderungen; die entzündlichen Erscheinungen verschwinden im Innern des Knötchens, während sie an seiner Peripherie spärlicher werden. Hier sind nur dünne Streifen jungen Bindegewebes sichtbar, die sich um das Knötchen anordnen, wo die fibroblastische Proliferation deutlich ist.

Diese hat aber noch nicht zur Bildung robuster Bindegewebslagen geführt.

In den Räumen zwischen den tuberkulösen Herden nur spärliche Büscheln elastischen Gewebes.

Zwölfter Fall. Lup us vulgaris tumidus nuchae. Hautstückchen aus einer talergroßen Effloreszenz, welche sukzessiv fünfmal je eine Stunde bestrablt wurde.

Biopsie: Zwanzig Stunden nach der letzten Applikation.

Makroskopisch: An der Applikationsstelle ist die Haut stark gerötet, dolent und mit einer kleinen gelblichen Kruste bedeckt.

Histologische Untersuchung. Die Alterationen sind außer geringen Intensitätsdifferenzen jenen des vorangegangenen Falles gleich.

Man bemerkt jedoch, dab, wenn an einer und derselben Stelle verschiedene Applikationen, sukzessiveiner nach der anderengemacht wurden, die Erscheinungen von Nekrose im epidermo-papillaren Teile schon seit der ersten Zeit nach der Applikation viel mehr, dagegen jene von Exsudation weniger akzentuiert sind.

Dreizehnter Fall. L u p s vulgaris diffusus. Hantstückchen aus einer lupösen Effloreszenz aus der suprahyoidalen Gegend, die sukzessiv durch je 40 Minuten dreimal bestrahlt wurde.

Biopsie: Zehn Tage nach der letzten Applikation.

Makroskopisch: Der nach der durch die Applikation hervorgerufenen Blase sukzessive Substanzverlust vollständig geheilt. Die Effloreszenz ist modifiziert, weniger infiltriert, flacher und mit glatter Haut bedeckt.

Histologische Untersuchung. Die Epidermis präsentiert die gewöhnliche Hypertrophie mit Hyperkeratose und Akanthose. In den 
oberen Lagen der Cutis, wo im geringen Grade die Ektasie der Gefäße, das Ödem und die Hämorrhagien vorkommen, ist eine sehr spärliche, aber diffuse Proliferation von Fibroblasten, besonders um die Gefäße, wo die Bindegewebszellen mit den anderen gewöhnlichen Elementen des Exsudates gemischt sind.

Diese Proliferation hört in den tieferen Lagen, an den Seiten und unterhalb des granulomatösen Gewebes auf, von welchen lange Reiben von Plasmazellen ausgehen, die längs der dilatierten Blut- und Lymphgefäße gegen das subkutane Bindegewebe ziehen.

Vierzehnter Fall. Lupus vulgaris diffusus. Hautstückchen aus einer Iupösen Effloreszenz des Vorderarmes, welche in fast vier Monaten siebenmal je eine Stunde bestrahlt wurde.

Biopsie: Zwanzig Tage nach der letzten Applikation.

Makroskopisch: Die sicher modifizierte Effloreszenz ist nicht mehr über die umliegende Haut erhaben. Mit dem Diaphanoskop sieht man noch hirsekorngrobe gelbe Punkte.

Histologische Untersuchung. Gewöhnliche Hypertrophie der Epidermis. In der Cutis ist der Papillarkörper noch merklich ödematös. Zwischen den noch aufgeblähten Bindegewebsfasern ist noch eine diffuse zellige Infiltration, bestehend, wie gewöhnlich, aus Erythrocyten, polynukleären Leukocyten, kleinen Rundzellen und jungen Bindegewebsbündeln in verschiedenen Stadien der Entwicklung. Die BlutgefäBe sind alle noch dilatiert und enthalten in ihrem Lumen zahlreiche, an die Wandungen haftende polynukleäre Leukocyten. Unter dem Papillarkörper der Cutis wird die Infiltration weniger intensiv und deutlicher fibroblastisch, bis man einem jungen schon organisierten Bindegewebe begegnet, das rund herum und in der Höhe die verschiedenen tuberkulösen Herde umgibt. In den letzteren sind noch die typische Struktur und die wenig oder gar nicht alterierten, von den epitheloiden und lymphoiden Zellen umgebenen Riesenzellen erhalten. An ihrer Peripherie sind deutliche Erscheinungen von gewöhnlicher Entzündung (Dilatation der GefäBe und Leukocytenexsudation) und bindegewebiger Proliferation. Tatsächlich dringen Fibroblasten mit ihrem verlängerten Kerne zwischen den granulomatösen Zellen ein.

In den tieferen Lagen der Cutis persistieren noch weniger akzentuiert das Ödem nnd die Dilatation der Gefäße.

Das elastische Gewebe kommt in irregulären Büscheln nur in den tieferen Lagen der Cutis vor; dagegen fehlt es in den höheren Lagen auch dort wo keine Spuren tuberkulöser Infiltration vorhanden sind.

Die Fasern sind größtenteils fragmentiert und gewunden.

Fünfzehnter Fall. L upus vulgaris cum tuberculis distinctis. Hautstückchen aus einem Tuberkel, dab viermal je 45 Minuten bestrahlt wurde.

Biopsie: Dreibig Tage nach der letzten Applikation.

Makroskopisoh: Das Tuberkel ist in seinem Volnmen reduziert, weniger angeschwollen und weniger das Niveau der umliegenden Haut überragend. 
Histologische Untersuchung. In der hypertrophischen Epidermis die gewöhnlichen Erscheinungen geringer Parakeratose und akzentuierter Akanthose.

In den oberen Lagen der $\mathrm{Cutis}$ persistieren noch das Ödem, die Dilatation der Gefäße und die spärliche aber diffuse Infiltration mit den gewöhlichen $\mathrm{Charakteren.} \mathrm{Diese} \mathrm{ist} \mathrm{noch} \mathrm{in} \mathrm{das} \mathrm{direkte} \mathrm{und} \mathrm{kompakte} \mathrm{Knöt-}$ chen eingedrungen, dessen $R i$ esenzellenwenig odergar nicht alteriert sind. Die Proliferationserscheinungensind aber spärlich und überschreiten, in der Tiefe, nicht das untere Niveau des Tuberkelknötchens. Unter derselben in den tiefen Lagen der Cutis sind Anhäufungen perivasalen Exsudates.

Keine deutlichen Veränderungen der Drüs en außer einer geringen interlobulären Infiltration.

Das elastische $G$ ewebe fehlt vollständig in dem vom granulomatösen Knötchen eingenommenen Gebiete. Im übrigen ist dasselbe in unregelmäßigen Büscheln und die Fasern präsentieren deutliche Zeichen chemischer und struktureller Degeneration.

Sechzehnter Fall. Lupus vulgaris diffusus dorsi pedis. Hautstückchen aus einer Lupusefloreszenz, das allein seit sechs Monaten mit der Kromayerschen Lampe behandelt wurde. Es fanden zwölf Applikationen, mit Intervallen von fünfzehn Tagen zwischen den einzelnen, statt.

Biopsie: Dreißig Tage nach der letzten Applikation.

Makroskopisch: Die Effloreszenz ist weniger rot, weniger infiltriert, hat leicht narbiges Anssehen. Unter dem Drucke des Diaphanoskopes sieht man eine tiefe Infiltration and in deren Mitte gelbe Pünktchen.

Histologische Untersuchung. Die Epidermis wegen geringer Hyperkeratose und mehr akzentuirter Akanthose sehr hypertrophisch.

Der obere Teil der $\mathrm{Cu}$ tis wird von einem jungen Gewebe, neuer Bildung, mit dünnen, parallel geordneten Fibrillen eingenommen, die mit $\mathrm{Hansen}$ blabrosarot tingiert und mit oblungen fusiformen Kernen von Fibroblasten bestreut sind. Dieses junge Gewebe überschreitet in der Tiefe nicht die Grenzen des Papillarkörpers, obgleich hie und da an den Seiten des kleinen granulomatösen Knötchens spärliche Fibroblasten wahrgenommen werden. Dieses, in seinem Volumen reduziert, ist oben von dem Bande eines jungen Bindegewebes neuer Bildung wie komprimiert, aber an den Seiten und unten, wo im Bindegewebe geringe entzündliche Erscheinungen von Dilatation der Gefäße und von Exsudation persistieren, frei.

Das Knötchen hat dichtes und kompaktes Aussehen und besteht aus einem großen Haufen kleinen mononukleären Zellen und Plasmazellen außer aus einigen aus den dilatierten Gefüßen ausgetreten polynukleären Leukocyten. Mehr unten, gegen die tiefen Lagen der Cutis, sind zwei 
sehr schöne Exemplare von sehr voluminösen Riesenzellen mit körnigem Protoplasma und ihren an der Peripherie gelegenen Kernen, in denen es nicht möglich war, deutliche pathologische Veränderungen zu finden.

Im Innern des Knötchens lassen sich nicht, wiebeiden mit Finsen behandelten Fällen, Bindegewebsstreifensehen, welche in sein Innereseindringen und die Lockerung hervorrufen. Trotzder bedeutenden Zahl von Applikationen und der oberflächlichen Narbe hat das Knötchen frisches und kompaktes Aussehen und ist in seinem unteren Teile vollständig frei, sodaß es längs der Lymphgefäße Zellverzweigungen drängen kann.

Das unten und an den Seiten des Knötchens vorkommende elastische Gewebe fehlt vollständig im Narbengewebe.

Siebzehnter Fall. Lupus vulgaris cum tuberculis dis tinctis. Hautstückchen mit einem linsengroßen Tuberkel, das in dre: Monaten sechsmal je eine Stunde bestrahlt wurde.

Biopsie: Fünfundvierzig Tage nach der letzten Applikation.

Makroskopisch: Das Tuberkel ist in seinem Volumen reduziert. Während es früher eher angeschwollen war und das Niveau der Haut überragte, ist es jetzt welk wie in eine narbig aussehende und leicht pigmentierte Haut eingelagert.

Histologische Untersuchung. Die Epidermis, wie gewöhnlich, diskret verdickt, besonders die malpighische Keimschicht derselben. Die oberen Lagen der $\mathrm{Cu}$ tis werden gänzlich von einem jungen Bindegewebe eingenommen, das mit seinen fibrillären Streifen und parallel und longitudinal geordneten Kernen auffällt.

Dieses junge Gewebe bedeckt oben, und überschreitet kaum unten an den Seiten ein Tuberkelknötchen, das sich longitudinal durch einen guten Teil des Schnittes diffundiert. Dasselbe besteht aus zahlreichen, normal aussehenden, von einer dichten Inflitration von kleinen mononukleären Elementen und Plasmazellen umgebenen Riesenzellen; nur an der Peripherie des Knötchens, speziell am unteren Pole, sind noch einige spärlichen polynukleären Leukocyten und einige jungen Bindege webszellen. Solche Elemente finden sich nicht im inneren Teile des Knötchens, das ein frisches und kompaktes Aussehen behält und von der dasselbe umgebenden, unten aus normalem schlaffen Bindegewebe, oben aus jungem Bindegewebe neuer Bildung bestehenden Bindegewebsmasse deutlich abgegrenzt wird. Unterbalb des Knötchens und in den Präparaten, ị denen der Schnitt auf das Knötchen selbst gefallen ist, persistieren noch, obgleich nichtsehrakzentuiert, das ödem, die Dila tation der GefäBe und die perivasale Exsudation.

Das elastische Gewebe fehlt im granuromatösen Gewebe und ist nur in Form spärlichster zarter Fibrillen im jungen Narbengewebe vorhanden. 
Aehtzehnter Fall. Lupus vulgaris cum tuberculis distinctis. Hautstückchen mit einem unversehrten Tuberkel, das achtmal je eine Stunde belichtet wurde.

Biopsie: Vier Monate nach der letzten Applikation.

Makroskopiseh: Das Tuberkel ist viel weniger prominent und erscheit als ein kleiner, gelblicher, tief in eine leicht pigmentierte narbig aussehende Haut eingelagerter Punkt.

Histologische Untersuchung. An einigen Stellen der an Dicke kaum vermehrten Epidermis fehlen die Hornschicht und das Stratum lucidum; in der Keimschicht ein leichter Grad von Interzellularödem. In den oberen Lagen der $\mathrm{Cu}$ tis, unmittelbar unter der Epidermis noch ein geringer Grad von Ödem, Dilatation der Gefäße und kleine Anhäufungen perivasaler, besonders aus kleinen Rundzellen, einigen roten Blutkörperchen, einigen polynukleären Leukocyten und jungen Bindegewebselementen (Fibroblasten) bestehenden Infiltration.

Darunter findet sich eine Lage jungen Bindegewebes neuer Bildung mit seinen Bündeln und oblungierten longitudinal angeordneten Kernen. Diese Bindegewebslage überschreitet nach unten nicht die mittleren Lagen der Cutis und umgibt oben und an den Seiten in Form einer Callotte ein kleines im Zentrum gelegenes Knötchen, welches aus einer sehr kompakten Masse von Zellen, besonders von Lymphocyten und Plasmazellen besteht. Nur an der Peripherie des Knötchens um die dilatierten und mit Blut gefüllten Gefäße sind einige polynukleäre Leukocyten.

$E_{S}$ sind noch einige größtenteils merklich alterierte Riesenzellen sichtbar; sie präsentieren tatsächlich irreguläre Konturen. meistens chromatinarme, ordnungslos zerstreute Kerne und beginnende Vacuolisation im Zelleibe. Im Innern des Knötchens sind einige Fibroblasten, aber man nimmt nicht wirkliche Bindegewebslamellen wahr, die die Dicke des Knötchens selbst überschreiten.

Unter dem Knötchen erscheint das Bindegewebe wie normal als ein schlaffes Gewebe; die Drüsenknäuel sind nicht alteriert, sie zeigen nur einige Infiltrationszellen im interlobulären Bindegewebe.

Das elastische Gewebe ist in langen, welligen und ramifzierten Fasern im schlaffen Bindegewebe der tiefen Lagen der Catis; sie sind irregulär and ein wenig gewunden in den mittleren Lagen und fehlen fast vollstänđig in den oberen Lagen der Cutis entsprechend dem Narbengewebe.

\section{Allgemeine Schlußfolgerungen.}

Wenn wir den durch die Lichtapplikationen mit der Kromayerschen Lampe an der gesunden Haut hervorgerufenen histopathologischen Prozeß kurz wiederholen wollen, so finden wir, daß die einige Stunden nach der Applikation schon ichtbaren ersten Tatsachen Entzündungserscheinungen sind, dies 
von einer Dilatation der kleinen Blutgefäße der Cutis dargestellt sind, eine Dilatation, die um so intensiver wird, je mehr wir uns der Epidermis nähern.

$\mathrm{Zu}$ gleicher Zeit tritt ein diffuses Ödem auf, dessen Intensitätsmaximum auch entsprechend dem Papillarkörper liegt; die Gefäße füllen sich mit Blut, es beginnt die Diapedesis und es bildet sich das perivasale Exsudat von kleinen Rundzellen, Erythrocyten und reichlichen polynukleären Leukocyten. Inzwischen dehnt das Ödem die Epidermiszellen der Keimschicht, zwischen welchen die aus der Cutis kommenden verschiedenen Elemente des Infiltrates hineindringen. In der Cutis führt das Fortschreiten der Alterationen nur zu einer Vermebrung des Exsudates in den oberen Lagen, dagegen ist dasselbe in den tieferen Lagen, wo aber die Dilatation und Thrombose der Gefäße und die kleinen Hämorrhagien persistieren, viel spärlicher.

In der Epidermis dagegen nehmen die Alterationen rasch zu. Das intensiver gewordene Ödem lockert die Masse der Keimschicht, in der kleine, mit einer serösen gefüllte Räume entstehen. Sofort treten in den Stachelzellen Degenerationserscheinungen, trübe Degeneration, vacuoläre Degeneration und Chromatolyse auf; in der Keimschicht, unterhalb der Hornschicht bilden sich zahlreiche Blasen. Die Epidermiszellen fallen rasch der Nekrose anheim, bis die ganze Epidermis zu einer amorphen Leiste reduziert und durch das Ödem zu einer breiten Blase abgehoben wird, der als Unterlage der Papillarkörper dient.

Den raschen Destruktionserscheinungen der Epidermis folgen mit genügender Beschleunigung und Rapidität jene der Reparation. An den Rändern der nekrotisierten Epidermis gelangen die Epidermiszellen in ein Stadium lebhafter Proliferation und die neue Epidermis bildet sich wieder hypertrophisch in allen ihren Schichten mit Erscheinungen von Hyperkeratose, Parakeratose und Akanthose.

In der $\mathrm{Cu}$ tis dagegen entsteht der Regenerationsproze $\boldsymbol{B}$ viel später und derselbe ist ein langsamer. Die Entzündungserscheinungen, obgleich nicht sehr lebhaft, sind sehr hartnäckig. Die Exsudation verschwindet stufenweise, aber langsam, 
während das Ödem und die Gefäßerweiterung noch eine lange Zeit erhalten bleiben. Inzwischen erscheinen speziell in der Nähe der Gefäße junge Bindegewebszellen, welche sich rasch vermehren, bis die ganze Cutis zwischen den perivasalen Resten des Infiltrates eine sehr aktive Proliferation von Fibroblasten zeigt.

Gering und wenig bedeutend ist die Teilnahme der Anhängsorgane, Haarfollikel und Drüsen, der Haut an dem histopathologischen Prozesse.

Das elastische Gewebe dagegen erleidet am Anfange deutliche chemische und strukturelle Veränderungen, bis es schließlich in den oberen Lagen der Cutis fragmentiert und zerstört wird.

Die histologischen Veränderungen an der lupösen Haut sind im allgemeinen fast die gleichen; es kommen nämlich dieselben Erscheinungen von Degeneration und Nekrose in der Epidermis, dieselben Entzündungserscheinungen in der Cutis mit dem Intensitätsmaximum im Papillarkörper vor. Dennoch ist ein Unterschied zwischen den zwei Prozessen, der darin besteht, daß an der gesunden Haut die Reaktion lebhafter und tiefer ist. Um zuerst aus den Entzündungserscheinungen (Gefäßerweiterung, perivasale Exsudation) und dann aus denen der Regeneration zu urteilen, sind diese aktiver und vor allem mehr ausgebreitet in der gesunden Haut, in der die Entzündungsexsudation und die sukzessive Proliferation von Fibroblasten bis in die tieferen Lagen der Cutis reichen; dagegen kommt dies in der lupösen Haut seltener vor, als würde fast das granulomatöse Gewebe ein Hindernis für den chemisch-physikalischen Reiz des Lichtes bilden.

Diese Tatsache wird von der mehrmals gemachten Beobachtung bekräftigt, daß das granulomatöse Knötchen jenes ist, das früh oder spät weniger Alterationen des photoderma. titischen Prozesses erleidet.

Tatsächlich, obgleich man in seinem Innern, infolge der Lichtapplikationen, kleine, mit Blut gefüllte dilatierte Gefäße sieht, ist das Ödem kaum akzentuiert, die leukocytäre Exsudation sehr spärlich und fast immer auf die Peripherie des Knötchens beschränkt. Wenn letzteres die Haut überragt und die ganze 
Dicke der Cutis einnimmt, so zeigen die Riesenzellen der oberen Lagen einige Alterationen; sie sind von den sie umgebenden Zellen getrennt; ihre Konturen sind irregulär, ihre Kerne unregelmäßig geordnet, in deren Innern manchesmal Vacuolisationszeichen. Die Riesenzellen der tieferen Lagen präsentieren dagegen keine deutlichen pathologischen Veränderungen und die granulomatöse Infiltration bildet in ihrem Innern eine geschlossene und kompakte Zellmasse.

Auch von den Regenerationserscheinungen, auf die sich im Grunde der Heilungsprozeb beim Lupus stiitzt, erleidet das wirkliche und eigene Knötchen eine sehr geringe Wirkung; die bindegewebige Proliferation, mit ihrem Intensitätsmaximum entsprechend dem Papillarkörper, muß gewiß eine Kompression ausüben und relativ eine Destruktion der oberen Teile des Knötchens selbst hervorrufen, ohne aber ihn vollständig von allen Seiten zu umgeben, ohne in das Innere seiner Zusammensetzung Fasern und Narbenstränge zu senden.

Infolge dieses Mangels oder dieser Spärlichkeit von bindegewebiger fibroblastischer Proliferation in den tieferen Lagen der Cutis, bildet die photodermatische Reaktion bei den Formen von Lupus diffusus ein Hindernis für die Propagation der granulomatösen Infiltration längs der Gefäße, eine Propagation, welche die Diffusion, trotz der Behandlung und die Rezidiven, trotz des Auftretens von gaten Resultaten an der Oberfläche erklärt.

Wenn wir zwischen dem von der Kromayerschen Lampe und dem vom Finsen-Lichtbogen hervorgerufenen ProzeB an der Hand der von verschiedenen Forschern und speziell der von Gavazzeni in unserem Institute vorgenommenen Untersuchungen Vergleiche herstellen wollen, so sehen wir, daß die beiden, obgleich eventuell gleichen Prozesse merklich von einander sich bezüglich der Intensität, Dauer und Folge der Entzündung und sukzessiven Regenerationserscheinungen unterscheiden.

Tatsächlich bedingen die Lichtapplikationen mit der Kromayerschen Lampe das Maximum der Reaktion in der Epidermis und dem Papillarkörper, dagegen reichen die Veränderungen bei den Lichtapplikationen viel mehr in die Tiefe; 
die Dilatation der Gefäße und vor allem das Ödem erstrecken sich auf die ganze Cutis, in der oft eine solche Tension erreicht wird, daß mit seröser Flüssigkeit gefüllte Höhlen gebildet werden.

Dieser Untersehied in der Intensität des phlogistischen Ödems ist vor allem im Tuberkelknötchen ersichtlich, das durch das Kromayersche Licht geringste Veränderungen erleidet, dagegen infolge der Applikation des Fins en-Lichtes wie dilatiert und manchmal durch die Tension des Ödems zerrissen wird.

$\mathrm{Zu}$ gleicher Zeit sind reichliche Leukocyteninvasion und beschleunigte und intensive Degeneration der Riesenzellen vorhanden.

Die Veränderungen entzündlichen Charakters (Ödem, Dilatation der Gefäße und Exsudation) infolge der Applikationen mit Kromayer schem Lichte, obgleich weniger intensir und weniger ausgebreitet, sind dagegen von sehr langer Dauer; manchmal kann man mit Leichtigkeit, einige Monate nach der Applikation, die Dilatation der Gefäße und die kleinen Hämorrhagien persistieren sehen, während dieselben nach den Finsenapplikationen im allgemeinen schneller entschwinden und die reaktiven Regenerationserscheinungen beschleunigter und lebhafter nachfolgen. Natürlich auch bezüglich dieser kommen in beiden Prozessen markierte Differenzen vor, denen vor allem die Verschiedenheit des Erfolges bei der Lupusbehandlung zuzuschreiben ist.

Wie wir schon erwähnten, ist die Proliferation des Bindegewebes bei der mit dem Kromayerschen Lichte behandelten Haut fast ausschließlich auf den Papillarkörper beschränkt. Es kommt nicht wie nach den Finsenapplikationen eine aktive und auf die ganze Cutis ausgebreitete Neubildung so vor, daß das Tuberkelknötchen immer enger in einem Ringe eingeschlossen wird. In meinen Präparaten von durch längere Zeit mit der Kromaye r schen Lampe behandelten Fällen sieht man sehr gut, wie das fibröse Gewebe oberhalb des Knötchens, das unten ein normales schlaffes Bindegewebe besitzt, eine Calotte bildet.

Überdies können wir nicht, wenigstens in demselben Grade wie nach den Finsen applikationen, eine Invasion ron 
Fibroblasten in das Innere des Knötchens und sulzzessiv die Bildung von Bindegewebslamellen, welche es in jeder Richtung durchziehen und seine Zertrümmerung hervorrufen, wahrnehmen. Dagegen ist nach einer bedeutenden Zahl von Kromayerapplikationen das granulomatöse Knötchen sicher in seinem Volumen vermindert und an der Oberfläehe gequetscht, aber in seinem Innern immer von kompakten und normalen Aussehen und hat unten und an den Seiten freien Grang für seine Diffusion.

Auch in der Regeneration des elastischen Gewebes sind zwischen den zwei Prozessen markierte Differenzen.

Während in der Fin sen narbe die Reproduktion eines feinsten Retikulum elastischer Fasern, das der Narbe ihr Aussehen und ihre charakteristische Elastizität (Radaeli (24), Gavazzeni) (25) verleiht, charalteristisch ist, fehlt in der Kroma yerNarbe das elastische Gewebe immer in der Kutis oder es sind nur einige isolierte dünne Fasern vorhanden.

Diese Tatsache steht vielleicht in Beziehung mit der intensiven Nekrose, welche dieApplikation immer in diesem Hautniveau bedingt.

Welche Ursachen können eine solche Verschiedenheit in der Wirkung zwischen dem Finsenlichtbogen und der Kromayerschen Lampe bedingen? Unsere histologischen Resultate beweisen, daß die Oberflächenwirkngg der Kromayerschen Lampezuriel intensiv, die Tiefenwirkung dagegen zu wenig intensiv ist. Welchen Umständen kann man dies zuschreiben? Diese Frage können wir weder mit Sicherheit leicht beantworten, noch verfügen wir über die diesbezüglichen personellen Untersuchungen.

Auf Grund der von den früheren Experimentatoren erzielten Resultate, welche die chemisch-physikalischen und biologischen Eigenschaften des Kromayer-Lichtes in Beziehung mit dem Finsen untersucht haben, könnte man also vielleicht die Ursache in der Komposition des Lichtspektrums der Kromay e r schen Lampe suchen. Die Extension desselben ist tatsäcblich, obgleich es sehr reich an ultravioletten Strahlen, ist, sehr beschränkt und entbält sehr wenige von jenen Strahlen, die am meisten penetrierend sind.

Wir dürfen aber nicht vergessen zu berücksichtigen, von welchem Einflusse die Schwierigkeit ist, eine energische Kom- 
pression zu machen und sukzessiv eine Ischämie der Gewebe hervorzurufen, welche den Durchgang eines mit geringerer Intensität dotierten Lichtes erleichtere.

Auf Grund dieser histologischen Befunde, welche Schlußfolgerungen können wir im klinischen Sinne bezüglich der therapeutischen Indikationen ableiten?

Die klinische Untersuchung verschiedener Patienten, die wir unserer Beobachtung unterworfen, hat bewiesen, daB durch die Behandlung mit der $\mathrm{Kr}$ omayer schen Lampe immer eine sichere Modifikation in den verschiedenen Lupusformen erfolgt,

Die distinkten Tuberkel nehmen $a b$, werden weniger überragend, bis sie als kleine in die Haut eingelagerte Punkte erscheinen. Die Effloreszenzen werden weniger erhaben und weniger infiltriert.

Wir haben weder klinische noch histologische sichere Daten, um anzunehmen, ob eine definitive Heilung durch die prolungierte Kromayersche Behandlung möglich sei.

Im sechzehnten konsekutiv durch sechs Monate behandelten Falle war die Modifikation eine merkliche. aber nicht definitive wie eine entsprechende Effloreszenz, die gerade so lange mit $\mathrm{F}$ in senapplikationen behandelt wurde.

Aus der Gesamtheit der Untersuchungen können wir den Schluß ziehen, daß die Kromayersche Lampe sehr nützliche Anwendung in der Lupustherapie findet. Dieselbe eignet sich besonders für die hypertrophischen tuberösen Formen mit oberflächlichem Infiltrate, bei welchen sie als Vorbehandlung an Stelle der mit Roentgen treten kann. Sie kann aber nicht das $\mathrm{F}$ in s e $\mathrm{n}$ bogenlicht ersetzen, das immer von prompter, intensiver und viel sicherer Wirkung ist.

\section{Literatur.}

1. Kromayer. Quecksilberwasserlampen zur Behandlung von Haut und Schleimhaut. Deutsche med Wochenschr. 1906.

2. Wi chmann. Bemerkungen zu der Publikation des Herrn Prof. Kromayer: Quecksilberwasserlampen etc. Deutsche med. Wochenschr. 1906. pag. 688.

3. Schüler. Zur Frage der Wirkung von Quecksilberdampflampen. Deutsche med. Wochenschr. 1906. pag. 680 .

4. Kromayer. Uviollampe, Quarzlampe, Quecksilberwasserlicht. Deutsche med. Wochenschr. 1906. pag. 887.

5. Blaschko. Berliner medizinische Gesellschaft. 16. Jänner 1907. 
6. Kromayer. Die Anwendung des Lichtes in der Dermatologie. Berlin. Klinische Wochenschr. 1907.

7. Gunni Busck. Bemerkungen über die Kromayersche Quecksilberwasserlampe. Berl. Klin. Wochenschr. Juli 1907.

8. Wichmann. Experimentelle Untersuchungen über die biologische Tiefenwirkung des Lichtes der medizinischen Quarzlampe und des Finsenapparates. Münchn. med. Wochenschr. 1907. Nr. 28.

9. Hern Carl und Hesse. Experimentelle und klinische Untersuchungen über die Wirkungen des ultravioletten Lichtes. Dermatol. Zeitschrift. Bd. XIV. 1907.

10. Maas J. Die medizinische Quarzlampe, eine neve Lampe für Phototeraphie. Ref. in Münchn. med. Wochenschr. 1907. Nr. 41.

11. Pürckhauer. Experimentelle Untersuchungen über die Tiefenwirkung der Kromayerschen Quarzlampe an normaler Haut. Archiv für Dermatologie und Syphilis. Bd. LXXXVII. 1907.

12. Mulzer. Vergleichende experimentelle Untersuchungen über die Wirkung des Finsenschen Kohlenlichtes und der medizinischen Quarzlampe. Archiv f. Dermat. u. Syph. Bd. LXXXVIII. 1907.

13. Busck. Loc. cit.

14. Wetterer. Quelques observations sur l'action de la nouvelle lampe de Quarz au mercure. Archives d'électricité médicale. Maggio 1907.

15. Johansen. Untersuchung uber die Wirkung der Kromayerschen Lampe und der Finsen-Reynlampe auf Chlorsilberpapier. Berliner klin. Wochenschr. August 1907.

16. Behring. Über die Wirkung violetter und ultravioletter Lichtstrahlen. Medy. naten. Arch. 1907. Bd, I.

17. Bordier-Nogier. Recherches experimentales sur la lampe à vapeur de mercure et au Quarz. (Kromayer.) Archives d'électricité médicales. Maggio 1908.

18. Ma ar Wilhelm. Die Tiefenwirkung der Finsen-Reynlampe und der Kromayerlampe. Archiv f. Dermatol. u. Syph. Bd. XC. 1908.

19. Jansen Hans. Histologische Untersuchung der durch Kromayers Quecksilberquarzlampe erregten Lichtentzündung. Ibidem.

20. Jansen. Loc. cit.

21. Pellizzari. La Fototerapia. Relazione al Congresso internazionale di Fisioterapia. Roma. 1907.

22. Mazzoni. La Fototerapia colle lampade a vapore di Mercurio e specialmente colla lampa da Kromayer. Comunicazione fatta al Congresso di Idrologia Climatologia e Terapia fisica. 1908.

23. Gavazzeni. Ricerche sulle modificazioni istologiche determinate dalle applicazioni di Firsen nella pelle luposa. Giornale italiano malattie veneree e della pelle. I. 1907.

24. Radaeli. Nuove osservazioni sulla istologia patologica e sulla cura del sarcoma idiopatico multiplo emorragico della cute. Lo Sperimentale. 1906.

\section{Erklärung der Abbildungen auf Taf. V.}

Fig. 1. Hautstückchen 25 Tage nach der Applikation (s. Fall 4).

Fig. 2. Hautstückchen mit einem Tuberkel; 4 Monate nach der 8. Applikation (s. Fall 18).

Aus dem italienischen Manuskripte übersetzt von M.U.Dr. Costantino Curupi in Prag-Bagni di Telese. 

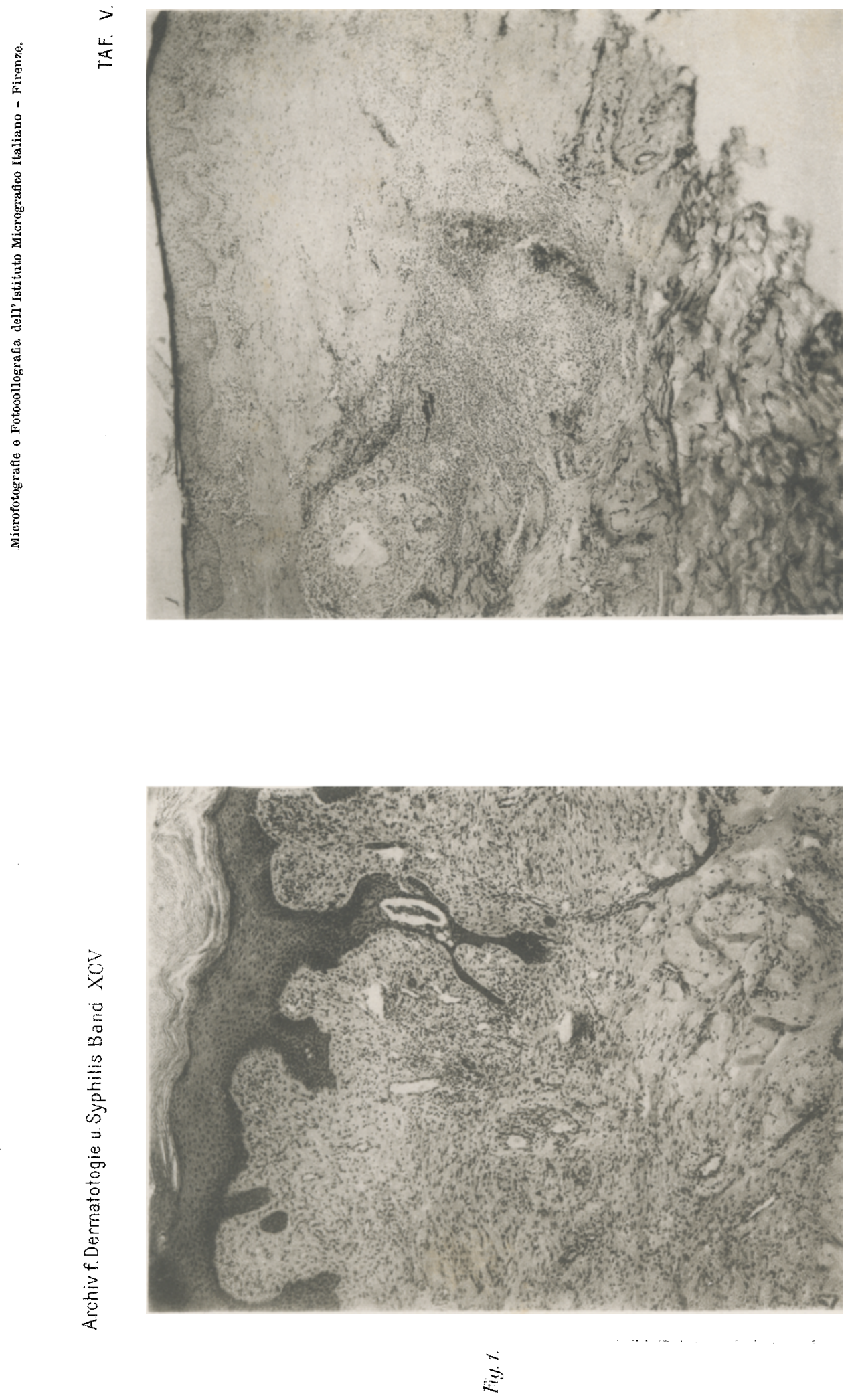

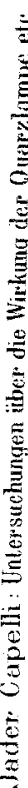

